\title{
Selective Tuning of the Blood Oxygenation Level-Dependent Response during Simple Target Detection Dissociates Human Frontoparietal Subregions
}

\author{
Adam Hampshire, John Duncan, and Adrian M. Owen \\ MRC Cognition and Brain Sciences Unit, Cambridge, Cambridgeshire CB2 7EF, United Kingdom
}

\begin{abstract}
Current models of working memory and focal attention converge on the idea of an adaptable global system, distributed across a network of frontal and parietal brain regions. Here, we examine how the human frontoparietal network selectively adapts to represent currently relevant information during a simple attentional task: monitoring for a target item in a series of nontargets. Across the entire frontoparietal network, there is selective response to targets, in line with a global system for coding task-relevant inputs. At the same time, there are striking dissociations in response to nontargets; whereas ventrolateral frontal cortex responds just to the target, more dorsal/anterior regions respond to all stimuli from the target category. The results show different degrees of target selectivity across different regions of the frontoparietal network.
\end{abstract}

Key words: attention; tuning; working memory; frontal cortex; parietal cortex; fMRI

\section{Introduction}

In the human brain, a common network of frontal and parietal regions is involved in a variety of tasks requiring the deliberate control of thought and action. In task-switching experiments, for example, the lateral prefrontal cortex (LPFC) and the posterior parietal cortex (PPC) commonly activate when the focus of attention is changed (Dove et al., 2000; Kimberg et al., 2000; Sohn et al., 2000; Dreher and Grafman, 2002; Braver et al., 2003; Hampshire and Owen, 2006). A similar network of brain regions activates during tasks requiring working memory and decision making (Duncan and Owen, 2000). This pattern of activation has led many researchers to propose that frontal and parietal brain regions form an adaptable global system, which underlies the flexibility of human behavior by enabling us to deliberately and selectively focus our attention on currently relevant information (Norman and Shallice, 1980; Dehaene et al., 1998; Duncan, 2001; Miller and Cohen, 2001). Highly adaptable frontal lobe activity has also been observed in nonhuman primates using single-cell recording (Miller and Cohen, 2001). During categorization tasks, for example, neurons in the monkey LPFC tune to respond selectively to just those categories that are currently relevant, while becoming unresponsive to those same categories when the task demands change (Freedman et al., 2001). More generally, neurons in the LPFC selectively respond to a broad range of taskrelevant information (e.g., stimuli, responses, rewards, and the

Received 0ct. 31, 2006; revised March 27, 2007; accepted March 28, 2007.

This work was supported by the Medical Research Council of the United Kingdom, and imaging data were acquired at the Wolfson Brain Imaging Centre at The University of Cambridge. We thank R. Thompson for his invaluable comments during the analysis.

Correspondence should be addressed to Dr. Adam Hampshire, MRC Cognition and Brain Sciences Unit, 15 Chaucer Road, Cambridge, Cambridgeshire CB2 2EF, UK. E-mail: adam.hampshire@mrc-cbu.cam.ac.uk.

DOI:10.1523/JNEUROSCI.0851-07.2007

Copyright $\odot 2007$ Society for Neuroscience $\quad$ 0270-6474/07/276219-05\$15.00/0 contents of working memory) (Duncan, 2001; Miller and Cohen, 2001), including learned target stimuli (Everling et al., 2002).

Here, we investigate the selective properties of the human attentional system. We use a simple monitoring task that enables us to examine how the frontoparietal network adapts to respond to the presentation of a target item among a series of nontargets. Nontargets are drawn from either the same or a different category as the current target item, allowing categorical similarity to be used as a metric for comparing how selective the neural response in different frontoparietal subregions actually is (Fig. 1). Using functional magnetic resonance imaging (fMRI), we search the brain for regions that follow either a tightly tuned profile, responding selectively to just the current target item, or that follow a more widely tuned profile, responding to all stimuli from the same category as that target. Even in this simple task, a broad swathe of frontoparietal cortex becomes involved in detection of the target item. Simultaneously, striking dissociations are apparent in the extent of selectivity in distinct subregions of this network.

\section{Materials and Methods}

Volunteers were instructed to look for a visually displayed target item within sequences of nontarget items. At the beginning of a sequence, the new target item appeared with the word "target" underneath for $2000 \mathrm{~ms}$. Subsequently, each item of the sequence was displayed for $750 \mathrm{~ms}$ (interstimulus intervals jittered from 250 to $750 \mathrm{~ms}$ ). At the end of each sequence, the question "Was the last stimulus the target?" appeared on the screen for $2500 \mathrm{~ms}$ and the volunteer was required to respond yes or no, using a button box with the first two digits of their right hand. The sequence length was varied from one to eight items in a row to ensure that attention was maintained throughout, and responses were made only when cued at the end of a sequence to keep critical contrasts free from motor activity. Fourteen right-handed volunteers between the ages of 20 and 40 undertook the fMRI task, which consisted of two 12 min blocks of 


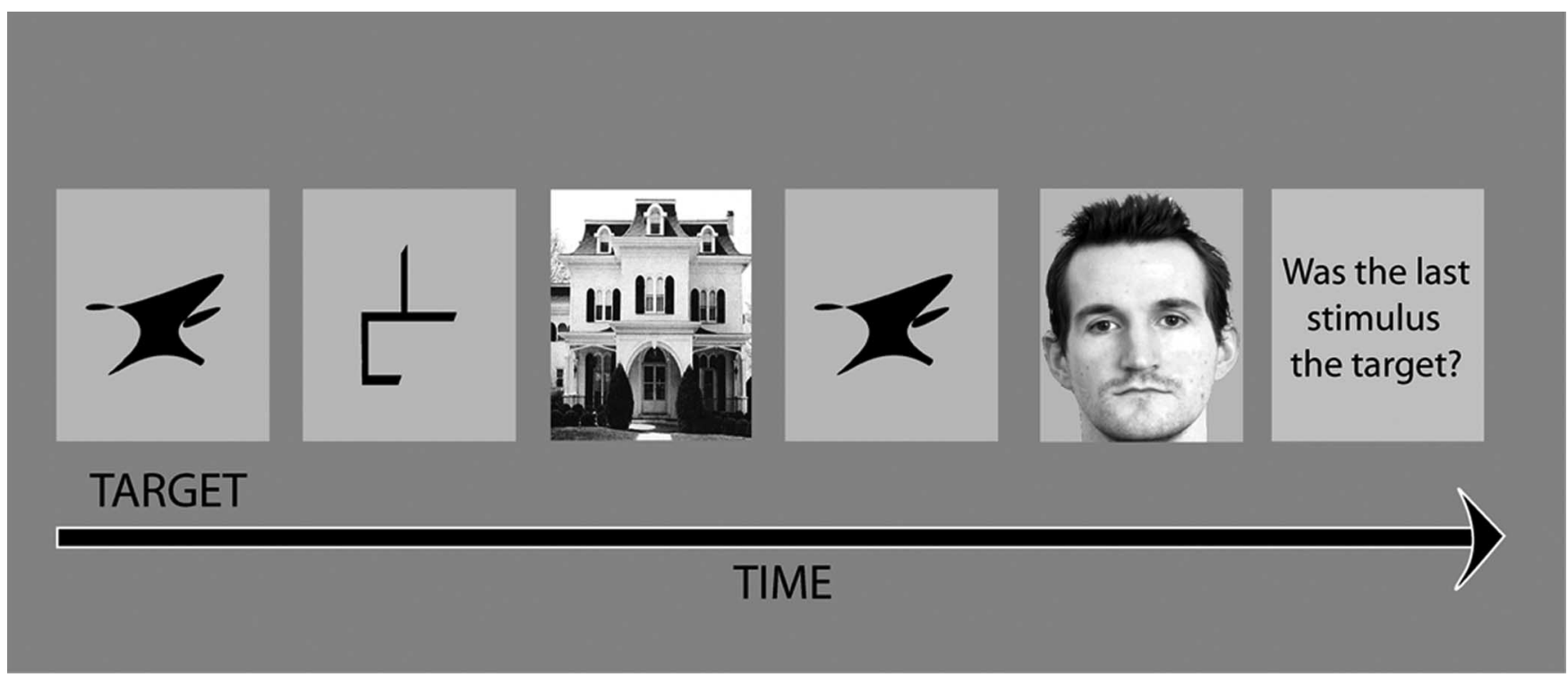

Figure 1. Task design. Volunteers looked for the presentation of a target item within sequences of visually displayed nontarget items. At the beginning of a sequence, the current target item appeared with the word "target." Sequence lengths were varied from one to eight stimuli in a row, and the target could be presented at multiple, any, or none of those positions. At the end of each sequence, the question "Was the last stimulus the target?" appeared on the screen, and the volunteer was required to respond yes or no, using a button box. Target and nontarget items were taken from a pool of stimuli, consisting of pictures from each of four distinct categories: faces, buildings, abstract line figures, and abstract shapes. The stimuli monitored could therefore be categorized according to whether they were the target item, nontargets from the same category as the target item, or nontargets from one of the other three categories.

scanning acquisition, each containing 40 stimulus sequences. Target and nontarget items were taken from the same pool of 80 unfamiliar stimuli, consisting of 20 pictures from each of four distinct categories: faces, buildings, abstract line figures, and abstract shapes. Volunteers monitored 376 stimuli over the two blocks combined, with 74 targets, 74 nontargets from the target category, and the remaining nontargets drawn from other categories. The sequences were predefined and pseudorandomized, with no individual picture used as the target item more than once within a block of scanning acquisition. Within a given sequence, the current target item could appear at any or multiple points. The presentation of targets, same-category nontargets, and other-category nontargets was balanced across the experimental block so that the relative probabilities were equivalent across all eight positions in the stimulus sequence.

Scanning was performed at the Wolfson Brain Imaging Centre ( 3 tesla Medspec s300 scanner; Bruker, Ettingen, Germany). Twenty-one $4 \mathrm{~mm}$ slices ( $1 \mathrm{~mm}$ interslice gap) were acquired in $1.1 \mathrm{~s}$ for each image (inplane resolution, $3.125 \times 3.125 \mathrm{~mm}$ ). Seven hundred twenty T2weighted echo-planar images (EPIs) depicting blood oxygenation leveldependent contrast were acquired per run, with the first 18 discarded to avoid T1 equilibrium effects. The experiment was programmed in Visual Basic, and the display projected onto a screen, visible from the scanner via a mirror, with stimuli subtending a visual angle of $6^{\circ}$.

Seventeen event types were included in the fMRI linear model, of which 12 were defined when the volunteer was monitoring for the target item. These were presentation of the following: the current target, nontargets from the target category, and nontargets from different categories, with these three conditions modeled separately for the four stimulus categories. The stage when the target was defined was also included in the fMRI model separately for each stimulus category, and the response cue formed the final event type.

Images were slice-time acquisition corrected, reoriented, subject motion corrected, geometrically undistorted using phase maps (Cusack et al., 2003), spatially normalized to the standard Montreal Neurological Institute EPI template, smoothed with an $8 \mathrm{~mm}$ full-width at halfmaximum Gaussian kernel, and modeled using Statistical Parametric Mapping 2 (SPM2; Wellcome Department of Cognitive Neurology, London, UK). The time series were high-pass filtered (cutoff period, $128 \mathrm{~s}$ ), and the hemodynamic response was modeled to the stimulus onsets and durations. The contrasts of interest were extracted, and the statistical maps for the critical contrasts were exported and examined in a randomeffects whole-brain analysis in SPM2.

In the group-level whole-brain analysis, we sorted target-sensitive regions into three types according to the extent of selectivity: tightly tuned, medium tuned, and widely tuned using a masked analysis. In each case, the main contrast used false discovery rate (FDR) correction at $p=0.001$, and masks were generated at $p<0.05$ uncorrected.

Tightly tuned brain regions were identified by contrasting presentation of the target with the mean of the two nontarget conditions. The mask excluded regions that were more responsive to nontargets from the target category than those from the other categories.

Regions that were medium tuned were also localized by using the contrast of targets against the mean of the two nontarget conditions. This time, the mask included only those regions that were more activated by nontargets from the target category compared with those from the other categories.

For widely tuned brain regions, the main contrast compared the mean of target and same-category nontargets with nontargets from other categories. The mask excluded brain regions that were more activated by the target than the same-category nontargets.

A second analysis used data from the second experimental block to define a series of regions of interest (ROIs). These ROIs were then used for analysis of the independent data from the first block. In block 2 data, comparison of targets with same-category nontargets generated peak activation bilaterally in the ventrolateral prefrontal cortex (VLPFC) at $p=0.05$ FDR corrected, and $10 \mathrm{~mm}$ spherical ROIs were centered on the peak coordinates (left: $x=-32, y=22, z=-6$; right: $x=46, y=18, z=$ 6). Comparison of target-category and other-category nontargets generated peaks in the right dorsolateral prefrontal cortex (DLPFC), right frontopolar cortex (FPC), and bilateral PPC, and $10 \mathrm{~mm}$ spherical ROIs were centered on the peak coordinates (DLPFC: $x=46, y=34, z=20$; FPC: $x=34, y=60, z=10$; left PPC: $x=-28, y=-60, z=42$; right PPC: $x=32, y=-60, z=48)$. There were no significant DLPFC or FPC activations in the left hemisphere for these contrasts even at the reduced threshold of $p=0.001$ uncorrected. Block 1 data were extracted from these ROIs for presentation of the target, same-category nontargets, and other-category nontargets using the MARSBAR ROI toolbox (Brett et al., 2002). Data were exported to SPSS (Chicago, IL) software for analysis using pairwise $t$ tests and ANOVA. 


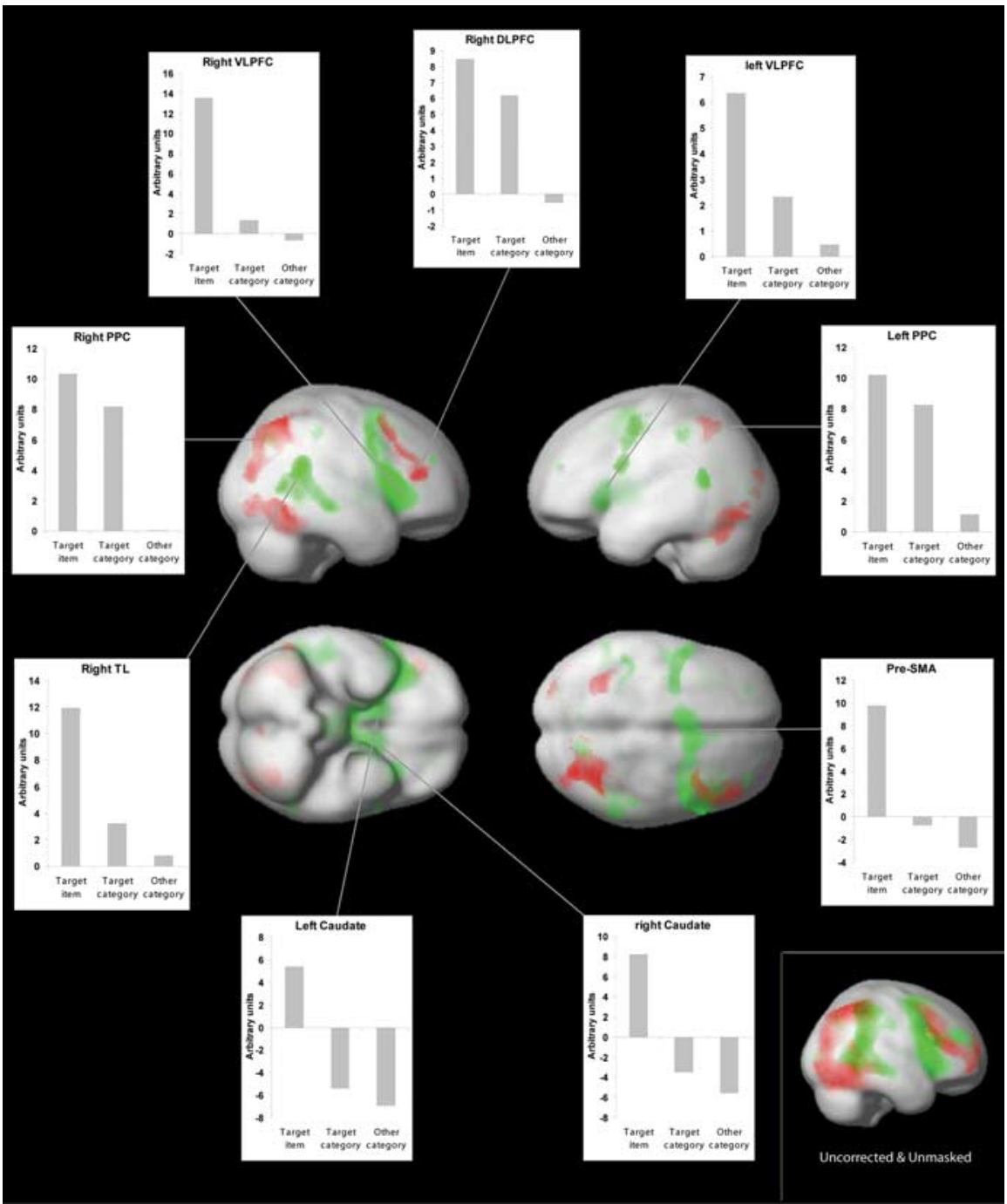

Figure 2. Relevance-sensitive brain areas. Results from the whole-brain analysis are shown. Green indicates brain regions that are tightly tuned to the target item; red indicates regions that are more widely tuned to the category level (see Introduction for definitions). The inset graphs display average activation levels extracted for the whole-activation clusters. Inset, Targets minus same-category nontargets and same-category nontargets minus nontargets from other categories, both contrasts at $p<0.05$ uncorrected and unmasked. Little overlap (yellow) is observed between the two activation patterns even at this low threshold. TL, Temporal lobe; SMA, supplementary motor area.

\section{Results}

In the whole-brain analysis, we first localized areas where activation was tightly tuned to the presentation of just the target stimulus, with no significant differences between responses to nontargets from the same or different categories as the target. Within the frontoparietal network, bilateral VLPFC and the presupplementary motor area followed a tightly tuned response function (Fig. 2 and supplemental Table 1, available at www.jneurosci.org as supplemental material).

A second contrast identified areas that followed an intermediate tuning pattern, because they were most activated by presentation of the target item but were also more activated by nontargets from the target category than nontargets from the other categories. Within the frontoparietal network, areas of significant activation were observed midway between the DLPFC and the VLPFC in the right hemisphere and bilaterally in the temporal-parietal junction (supplemental Table 1, available at www.jneurosci.org as supplemental material).

The third contrast localized widely tuned areas that responded to all stimuli from the current target category, regardless of whether it was the actual target that was presented. A region of activation was observed in the anterior right DLPFC extending toward the posterior extent of the right FPC. Bilateral PPC and bilateral ventral-stream visual areas also followed this widely tuned response function (Fig. 2 and supplemental Table 1, available at www.jneurosci.org as supplemental material).

A more focused ROI analysis confirmed the findings from the whole-brain analysis. Regions were defined based on peak coordinates from experimental block 2 , and the data were extracted from these ROIs for experimental block 1. Pairwise comparisons (Table 1) confirmed that the right DLPFC and PPC bilaterally were widely tuned, because both targets and nontargets from the target category generated more activation than nontargets from other categories, with no significant difference between the level of activation for targets and same-category nontargets. Conversely, the right VLPFC was tightly tuned, because activation was significantly greater for targets than nontargets, with no significant differences between the two nontarget conditions. In the right FPC, activation was only significantly greater for targets than other category nontargets.

For the right frontal ROIs, ANOVA confirmed a significant two-way interaction between ROI and similarity to the target $\left(F_{(4,52)}=9.49 ; p<0.001\right)$ with a significant main effect of similarity to the target $\left(F_{(2,26)}=15.61 ; p<0.001\right)$ and no significant main effect of ROI $\left(F_{(2,26)}=\right.$ $2.98 ; p=0.068)$. Different tuning patterns for different regions of the right prefrontal cortex are illustrated in Figure 3.

\section{Discussion}

The observation that a broad swathe of the frontal and parietal cortices becomes involved in simple target detection broadly supports models that propose a global system for attention and working memory (Norman and Shallice, 1980; Dehaene et al., 1998; Duncan, 2001; Miller and Cohen, 2001). Furthermore, the activation associated with detection of the target item is observed collapsed across the different stimulus categories, supporting the view that this global system has the capacity to code many different types of information (Duncan, 2001). However, in line with more modular accounts of frontoparietal organization, our findings demonstrate that even in a simple target detection task, subregions of this system can be dissociated by their differential responses to target category nontargets. Selectivity in some regions appears to extend to nontarget stimuli from the same category as the current target item, with the selective tuning curves widest in three distinct subregions: the more posterior extent of the left and right PPC and the anterior extent of the right DLPFC extending into the FPC.

The frontal activation foci reported in previous studies of 
Table 1. Pairwise comparisons from the ROI analysis

\begin{tabular}{llll}
\hline & $t$ & df & $p$ \\
\hline Left VLPFC & & & \\
$\quad$ Target vs target category & 3.5 & 13 & 0.004 \\
$\quad$ Target vs other category & 6.1 & 13 & 0.000 \\
$\quad$ Target category vs other category & 0.2 & 13 & 0.870 \\
Right VLPFC & & & \\
$\quad$ Target vs target category & 5.0 & 13 & 0.000 \\
$\quad$ Target vs other category & 8.6 & 13 & 0.000 \\
$\quad$ Target category vs other category & 0.1 & 13 & 0.927 \\
Right DLPFC & & & \\
$\quad$ Target vs target category & 1.8 & 13 & 0.095 \\
$\quad$ Target vs other category & 9.0 & 13 & 0.000 \\
$\quad$ Target category vs other category & 2.7 & 13 & 0.019 \\
Right FPC & & & \\
$\quad$ Target vs target category & 1.5 & 13 & 0.158 \\
$\quad$ Target vs other category & 6.0 & 13 & 0.000 \\
$\quad$ Target category vs other category & 1.8 & 13 & 0.091 \\
Left PPC & & & \\
$\quad$ Target vs target category & 0.7 & 13 & 0.520 \\
$\quad$ Target vs other category & 3.6 & 13 & 0.003 \\
$\quad$ Target category vs other category & 2.3 & 13 & 0.037 \\
Right PPC & & & \\
$\quad$ Target vs target category & 1.5 & 13 & 0.161 \\
$\quad$ Target vs other category & 6.9 & 13 & 0.000 \\
Target category vs other category & 3.8 & 13 & 0.002 \\
\hline
\end{tabular}

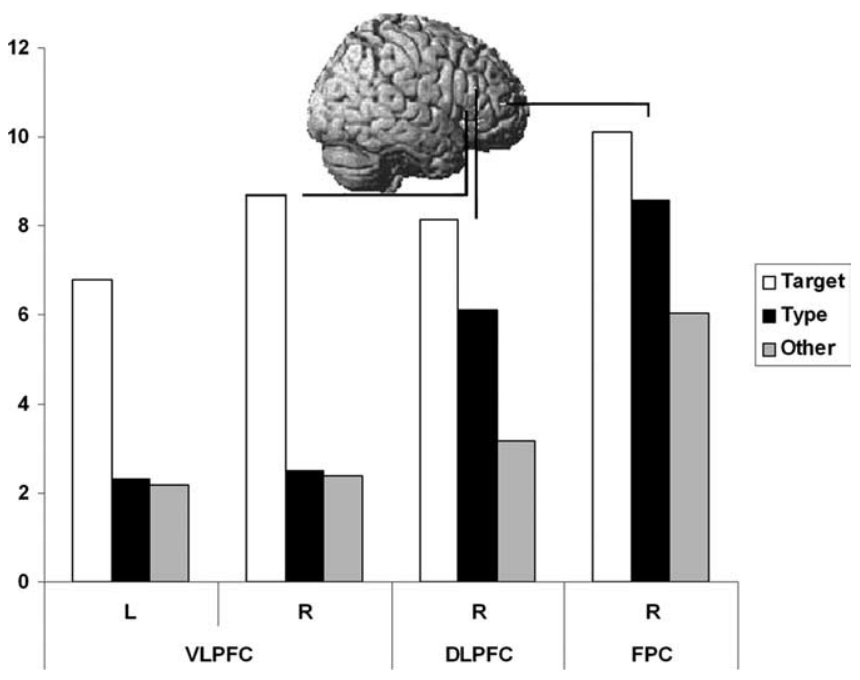

Figure 3. ROI analysis. Activation levels in the frontal cortex from the ROl analysis. There is a general trend of increased response to the nontarget items moving from VLPFC, through DLPFC to FPC. L, Left; R, right.

working memory and attention appear to conform to an overall anatomical hierarchy. The VLPFC has been implicated in the simplest of executive tasks, such as the intentional encoding and retrieval of memories (Dove et al., 2006) and the deliberate maintenance of items in working memory (Owen et al., 1990; Petrides 1994, 1995). In contrast, the DLPFC has been implicated in more complex tasks such as problem solving (Hampshire and Owen, 2006), the manipulation and monitoring of consciously maintained information (Petrides 1994, 1995; Owen et al., 1990; Petrides and Milner, 1982; D'Esposito and Postle, 1999), and the use of higher-level structure while maintaining multiple items in working memory (Bor et al., 2001). It has been suggested that the FPC is at the apex of this frontal hierarchy, because of its involvement in cognitive branching (delaying the execution of one task until completion of another) (Koechlin et al., 1999, 2003) and in combining multiple cognitive rules (Ramnani and Owen, 2004).

Previously proposed models that seek to account for these apparent specializations within the human frontal cortex can be broadly subdivided into those that propose differences in the types of information that are represented and those that propose differences in the processes performed. It is possible to account for our findings with either a representational or a process-based model.

The most literal interpretation of our results would propose that anatomically distinct frontoparietal subregions represent information at different levels of abstraction. So, although the ventral regions of the LPFC could represent concrete information (e.g., currently relevant objects and actions), the more dorsal/ anterior regions could represent more abstract goal-oriented information such as stimulus categories and task dimensions. This simple idea can also account for many findings from the broader literature. Performing more complex task demands requires the mental coding of more complex task programs. Often, this will involve the maintenance of information that is increasingly abstracted away from the specific material that is at the focus of attention, toward more general task parameters, such as relevant categories, rules, and subgoals. It seems possible, therefore, that the observed widening in selective tuning across the DLPFC and the FPC is caused by representation of task-relevant information at more abstract levels. This account is also reminiscent of previous representation-based models of human frontal differentiation (Wood and Grafman, 2003) and fits particularly well with O'Reilly et al. (2002), who proposed in their connectionist account that "prefrontal cortex areas are organized according to different levels of abstraction" as opposed to differences in process. In the model of O'Reilly et al. (2002), different frontal regions were specifically organized according to whether they coded for individual objects or abstract object dimensions (color and shape), an account requiring little extension to include abstract object categories.

An alternative hypothesis capable of explaining the current findings would propose two partially overlapping patterns of activity associated with two distinct cognitive processes. One of the patterns, related to the process of selectively recognizing currently relevant information, would be most strongly represented in the VLPFC and would be activated only at the point of target presentation. The other pattern, related to increased attentional demand, would be most strongly represented in the more dorsal and anterior regions of the frontal cortex. It seems possible that when the current item is not in the target category, it can be quickly rejected, whereas the smaller differences between targets and same-category nontargets requires the deliberate focusing of attention, thereby activating this attentional engagement pattern.

The observation of a similar gradient in the selective tuning curve in the PPC could also be explained in terms of representational or process-based differences. It seems likely, however, that this gradient simply reflects the direction of information flow in the posterior brain, with increased attentional modulation as information progresses from early visual areas along the dorsal visual stream toward motor control regions. In the frontal cortex, however, the concept of increasingly tight tuning with increasingly processed information is too simple to explain findings from the broader literature that indicate that the more widely tuned frontal subregions are typically implicated during the most complex of cognitive demands.

Clear dissociations have been hard to find between anatomically distinct components of the frontoparietal system and have 
historically tended to depend on complex differences in cognitive demand (e.g., maintaining, manipulating, or monitoring information in working memory). In this respect, the observation of a clear dissociation between the extent of selective tuning in frontoparietal subregions during simple target detection provides a valuable new insight into the nature of frontoparietal specialization.

\section{References}

Bor D, Duncan J, Owen AM (2001) The role of spatial configuration in tests of working memory explored with functional neuroimaging. Scand J Psychol 42:217-224.

Braver TS, Reynolds JR, Donaldson DI (2003) Neural mechanisms of transient and sustained cognitive control during task switching. Neuron 39:713-726.

Brett M, Anton J, Valabregue R, Poline J (2002) Region of interest analysis using an SPM toolbox. Paper presented at the Eighth International Conference on Functional Mapping of the Human Brain, Sendai, Japan, June.

Cusack R, Brett M, Osswald K (2003) An evaluation of the use of magnetic field maps to undistort echo-planar images. NeuroImage 18:127-142.

Dehaene S, Kerszberg M, Changeux J (1998) A neuronal model of a global workspace in effortful cognitive tasks. Neurobiology 95:14529-14534.

D'Esposito M, Postle BR (1999) The dependence of span and delayedresponse performance on prefrontal cortex. Neuropsychologia 37:1303-1315.

Dove A, Pollmann S, Schubert T, Wiggins CJ, Von Cramon DY, Yvesa D (2000) Prefrontal cortex activation in task switching: an event-related fMRI study. Brain Res Cogn Brain Res 9:103-109.

Dove A, Brett M, Cusack R, Owen AM (2006) Dissociable contributions of the mid-ventrolateral frontal cortex and the medial temporal-lobe system to human memory. NeuroImage 31:1790-801.

Dreher JC, Grafman J (2002) Dissociating the roles of the rostral anterior cingulate and the lateral prefrontal cortices in performing two tasks simultaneously or successively. Cereb Cortex 13:329-339.

Duncan J (2001) An adaptive coding model of neural function in prefrontal cortex. Nat Rev Neurosci 2:820-829.

Duncan J, Owen AM (2000) Common regions of the human frontal lobe recruited by diverse cognitive demands. Trends Neurosci 23:475-483.

Everling S, Tinsley J, Gaffan D, Duncan J (2002) Filtering of neural signals by focused attention in the monkey prefrontal cortex. Nat Neurosci 5:671676.

Freedman DJ, Riesenhuber M, Poggio T, Miller EK (2001) Categorical representation of visual stimuli in the primate prefrontal cortex. Science 291:312-316.

Hampshire A, Owen AM (2006) Fractionating attentional control using event related fMRI. Cereb Cortex 16:1679-1689.

Kimberg DY, Aguirre GK, D'Esposito MK (2000) Modulation of taskrelated neural activity in task switching: an fMRI study. Brain Res Cogn Brain Res 10:189-196.

Koechlin E, Basso G, Pietrini P, Panzer S, Grafman J (1999) The role of the anterior prefrontal cortex in human cognition Nature 399:148-151.

Koechlin E, Ody C, Kouneiher F (2003) The architecture of cognitive control in the human prefrontal cortex. Science 302:1181-1185.

Miller EK, Cohen JD (2001) An integrative theory of prefrontal cortex function. Annu Rev Neurosci 24:167-202.

Norman DA, Shallice T (1980) Attention to action: willed and automatic control of behavior (report no. 8006). La Jolla, CA: University of California, San Diego.

O’Reilly RC, Noelle DC, Braver TS, Cohen JD (2002) Prefrontal cortex in dynamic categorization tasks: representational organization and neuromodulatory control. Cereb Cortex 12:246-257.

Owen AM, Downes JJ, Sahakian BJ, Polkey CE, Robbins TW (1990) Planning and spatial working memory following frontal lobe lesions in man. Neuropsychologia 28:1021-1034.

Petrides M (1994) Frontal lobes and working memory: evidence from investigations of the effects of cortical excisions in nonhuman primates. In: Handbook of neuropsychology, Vol 9 (Boller F, Grafman, J, eds), pp 59-82. Amsterdam: Elsevier.

Petrides M (1995) Impairments on non-spatial self-ordered and externally ordered working memory tasks after lesions of the mid-dorsal part of the lateral frontal cortex in the monkey. J Neurosci 15:359-375.

Petrides M, Milner B (1982) Deficits on subject-ordered tasks after frontal and temporal lobe lesions in man. Neuropsychologia 20:249-262.

Ramnani N, Owen AM (2004) Anterior prefrontal cortex: insights into function from anatomy and neuroimaging. Nat Rev Neurosci 5:184-94.

Sohn MH, Ursu S, Anderson JR, Strenger VA, Carter CS (2000) The role of prefrontal cortex and posterior parietal cortex in task switching. Proc Natl Acad Sci USA 97:13448-13453.

Wood JN, Grafman J (2003) Human prefrontal cortex: processing and representational perspectives, Nat Rev Neurosci 4:139-147. 